Full length article

\title{
Low temperature selective growth of GaN single crystals on pre-patterned Si substrates
}

\author{
Jindřich Mach ${ }^{\mathrm{a}, \mathrm{b}}$, , Jakub Piastek ${ }^{\mathrm{a}, \mathrm{b}}$, Jaroslav Maniš a, Vojtěch Čalkovský a , Tomáš Šamořil a, b, \\ Jana Damková ${ }^{\mathrm{b}}$, Miroslav Bartošík ${ }^{\mathrm{a}, \mathrm{b}}$, Stanislav Voborný a, b, Martin Konečný ${ }^{\mathrm{a}, \mathrm{b}}$, Tomáš Šikola a, b \\ a CEITEC BUT, Brno University of Technology, Technická 3058/10, 61600 Brno, Czech Republic \\ ${ }^{\mathrm{b}}$ Institute of Physical Engineering, Brno University of Technology, Technická 2, 61669 Brno, Czech Republic
}

\section{A R T I CLE INFO}

\section{Keywords:}

GaN

Deposition

Selective growth

Low energy ions

Nanocrystals

Photoluminescence

\begin{abstract}
A B S T R A C T
We report on a hybrid method for fabrication of arrays of GaN nanocrystals by low-temperature UHV selective growth on pre-patterned silicon substrates covered by native oxide. Patterning of the substrates was performed by using a gallium focused ion beam (FIB). To get GaN nanocrystals at specific positions, Ga droplets were created at FIB patterned sites by evaporation of $\mathrm{Ga}$ atoms at $280^{\circ} \mathrm{C}$ substrate temperature first, and then modified by their post-nitridation using an ultra-low energy $(50 \mathrm{eV})$ nitrogen ion-beam at a sample temperature of $200^{\circ} \mathrm{C}$. To get larger arrays of GaN nanocrystals $(\approx 150 \mathrm{~nm}$ and $200 \mathrm{~nm}$ in diameter), such a sequential process was repeated in several cycles at slightly modified operation conditions. The quality of the nanocrystals was checked by measurement of their photoluminescence properties which proved the occurrence of the peak of a band edge emission at around $367 \mathrm{~nm}(3.38 \mathrm{eV})$.
\end{abstract}

\section{Introduction}

Organization and positioning of nanoparticles (NPs) and nanowires on semiconductor substrates represent a big challenge to take an advantage of their properties for fabrication of innovative integrated devices [1-3]. While their self-organization on naturally occurring patterns [4] or through different chemical interactions [5] provides high quality of nanocrystals at an ultra-high density $\left(>10^{10} \mathrm{~cm}^{-2}\right)$ over large scale surfaces, the array parameters (pattern shape, periodicity) are generally controlled by the material/substrate system and thus the degrees of freedom in their choice are rather limited [6,7]. The main limitations of this method are a lack of control over positioning of individual nanoparticles and thus a poor uniformity of the formed NPs ensemble.

Contrary to that, in fabrication methods of NP arrays grown on artificially pre-patterned substrates one can control the absolute position of dots over a long-range scale. The nucleation sites (e.g. holes, grooves or other features) on substrates can be prepared by various nanofabrication techniques such as electron-beam lithography (EBL), focused ion beam (FIB), nanosphere lithography, anodic aluminum oxidation (AAO), AFM patterning, nanoimprint lithography, etc. [8-13].

In the last decade, many efforts have been put into growth of ordered arrays of III-V quantum nanostructures semiconductors, espe- cially GaAs, InAs, and InP [14-17]. Naturally, growth of group III-nitride nanostructures has also attracted a lot of interest, but mostly ensembles of nanodots and nanocrystals distributed in disorderly have been achieved. One of the reasons for this is that the synthesis of GaN involves high activation energies generally overcome by high process temperatures $\left(\geq 600^{\circ} \mathrm{C}\right)$ which is associated with techniques such as metal-organic vapour-phase epitaxy (MOVPE) [18], plasma-assisted molecular beam epitaxy (PAMBE) [19] and chemical beam epitaxy (CBE) [20]. As a result, the spectrum of methods enabling selective growth of GaN nanostructures is limited as they must meet additional restraints related to this guided growth. For instance, in Ref. [21] the formation of ordered arrays of GaN microcrystals was studied. The microcrystals were grown by Ga droplet epitaxy at $500^{\circ} \mathrm{C}$ on As-terminated $\mathrm{Si}\left(\begin{array}{lll}1 & 0 & 0\end{array}\right)$ substrates patterned by low-energy $\mathrm{Ga}$ focused ion beam followed by nitridation at $600{ }^{\circ} \mathrm{C}$ using atomic nitrogen radicals generated by an RF radical cell.

However, in all these experiments high substrate temperatures during deposition were used $\left(600-1000^{\circ} \mathrm{C}\right)$ which can have a detrimental effect on some more complex structures and devices, being thus not compatible with some technology constraints laid on related fabrications steps.

To synthesize GaN films at lower temperatures $\left(\approx 300^{\circ} \mathrm{C}\right)$, a combination of $\mathrm{Ga}$ atomic beam evaporation (in principle an identical method to molecular beam epitaxy - MBE) and direct ion beam deposi-

\footnotetext{
* Corresponding author at: Institute of Physical Engineering, Brno University of Technology, Technická 2, 61669 Brno, Czech Republic.

Email addresses: jindrich.mach@centrum.cz (J. Mach); sikola@fme.vutbr.cz (T. Šikola)
} 


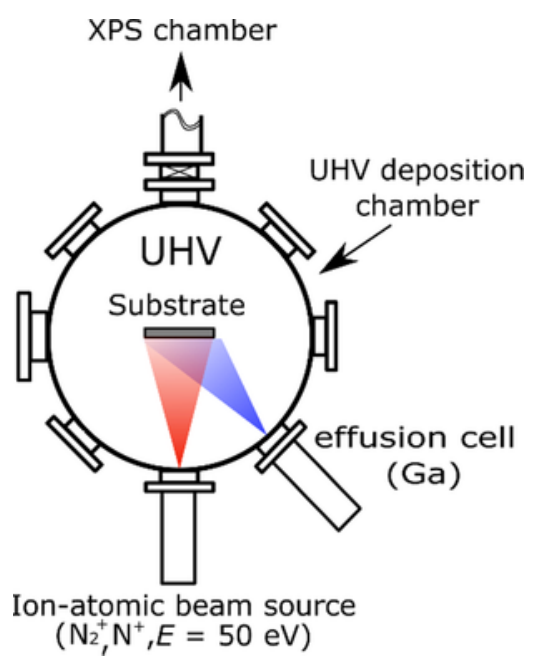

Fig. 1. Schematic of the deposition chamber. tion have been successfully used in our group [22]. In this method, similarly to [23], nitrogen ion beams of hyperthermal energies (below $100 \mathrm{eV}$ ) provide activation energy needed for synthesis of GaN and so lower substrate temperatures can be used. An important advantage of the combination of the direct Ion beam deposition and "classical" MBE technique over MOCVD is the mutual independence of both processes and compatibility with UHV conditions enabling application of a wider spectrum of in-situ analytical methods. Deposition of nitrogen and gallium can be carried out simultaneously or one after another. This fact together with UHV conditions can be beneficial for a study of the early stages of thin film growth. Individual stages of the deposition process can be synchronized in different ways or separated in time (e. g. alternating atom and ion beam fluxes) and in situ analyzed. On the other hand, the exact mechanism of growth of nitrides by this combined technique is not fully known as the irradiation with hyperthermal ions brings the system into a condition far from thermodynamic equilibrium. The knowledge of this growth mechanism is particularly important for preparation of GaN single crystals and their selective growth.

In this paper, we demonstrate how to utilize such a method for low temperature $\left(T<300^{\circ} \mathrm{C}\right)$ growth of arrays of $\mathrm{GaN}$ nanocrystals on $\mathrm{Si}$ substrates covered by native oxide. Ordered arrays of nanocrystals will
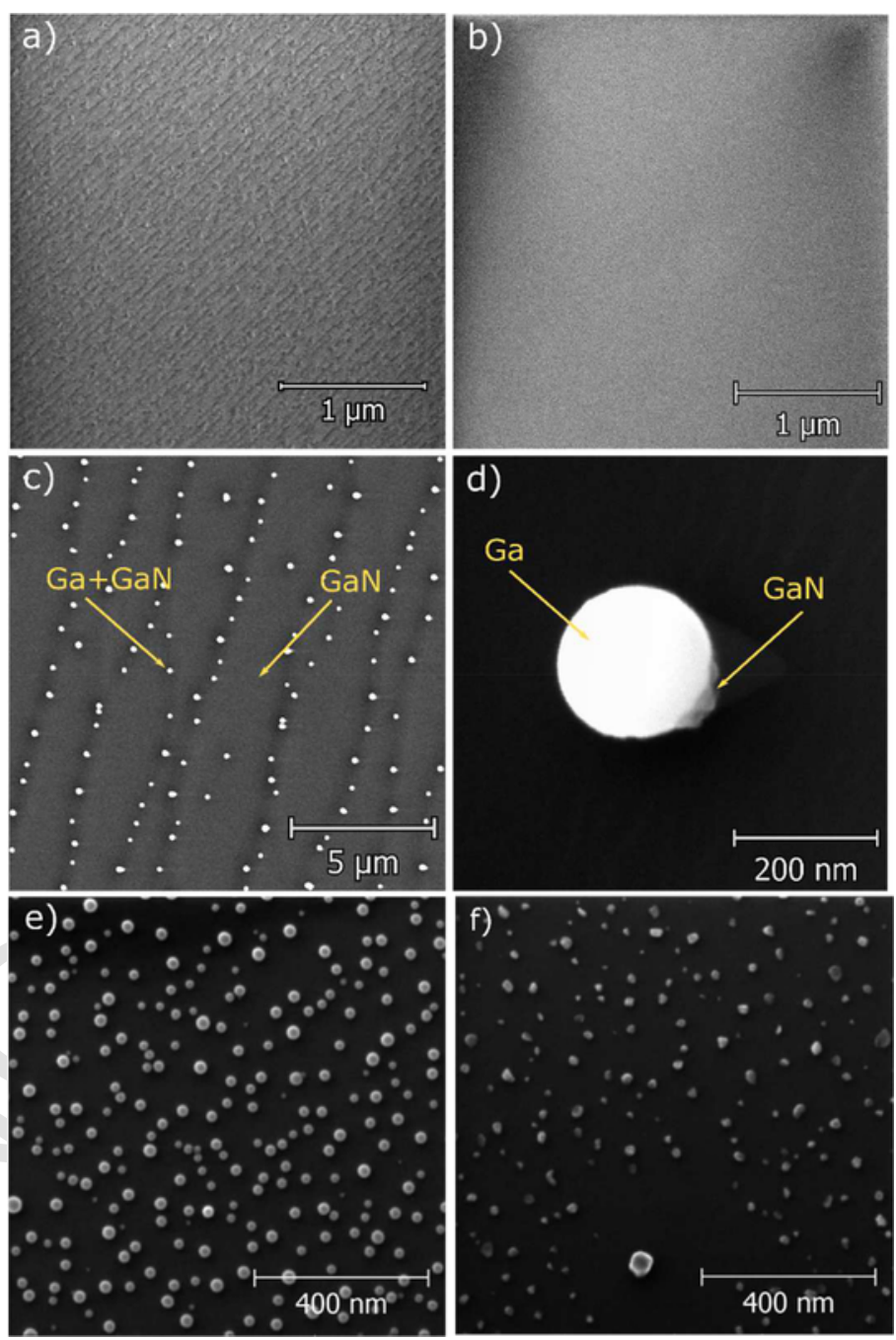

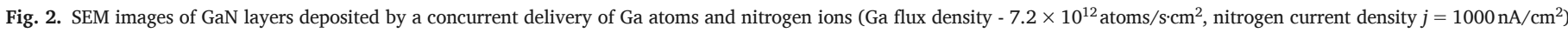

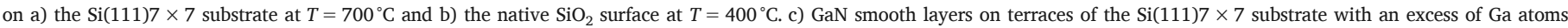

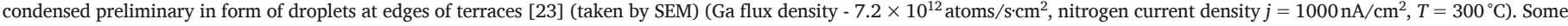

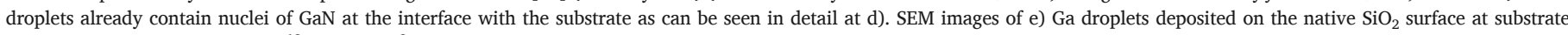

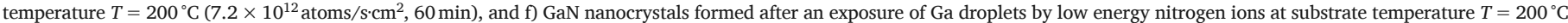
(nitrogen current density $j=1000 \mathrm{nA} / \mathrm{cm}^{2}, 60 \mathrm{~min}$ ). 


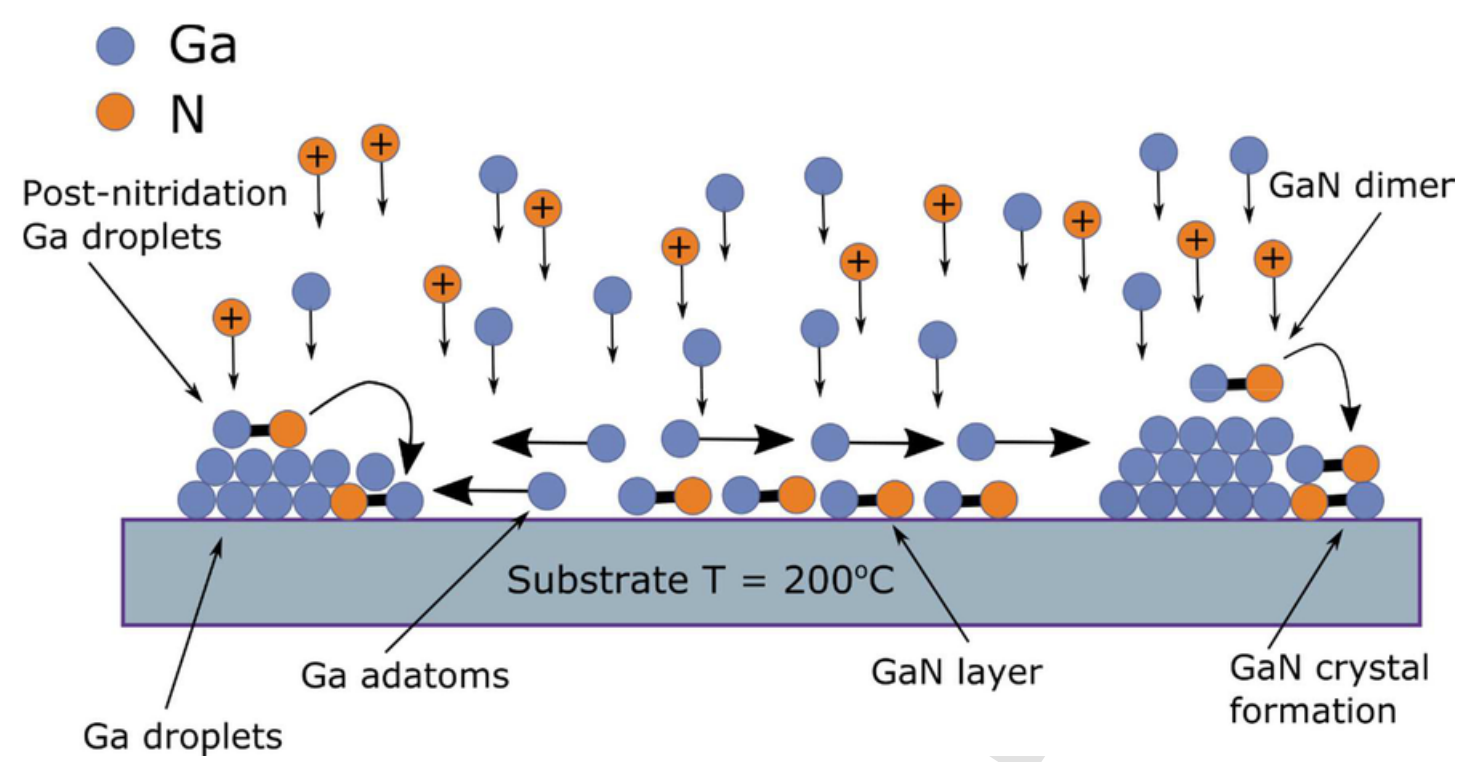

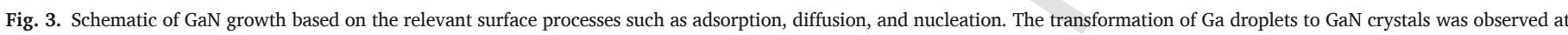
higher substrate temperatures $\left(T>200^{\circ} \mathrm{C}\right)$. This process is related to diffusion of GaN dimers on Ga droplet surface or inside of Ga droplets.
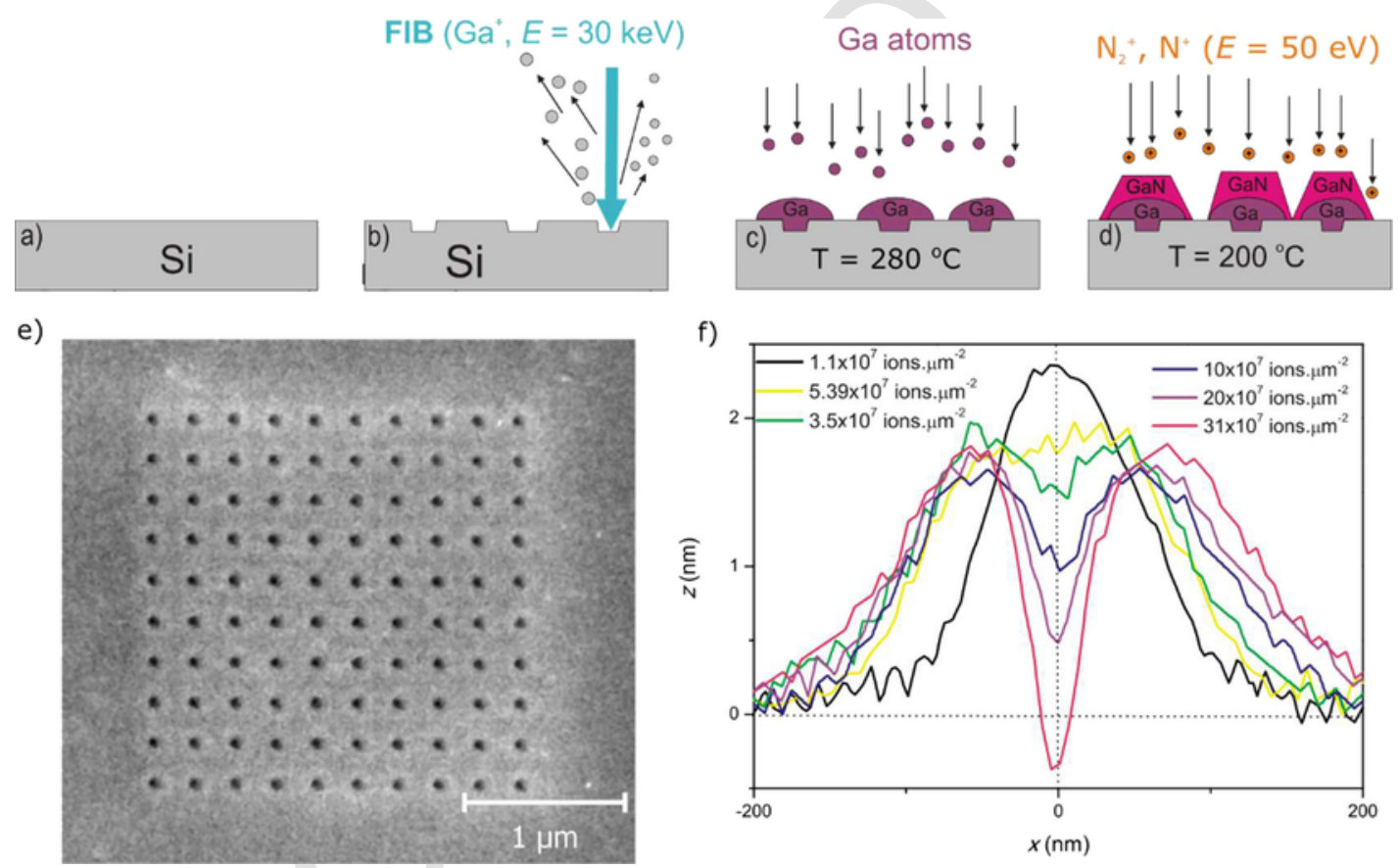

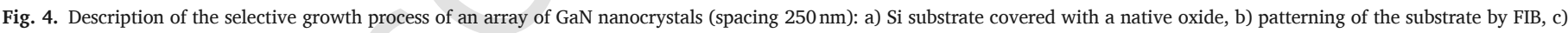

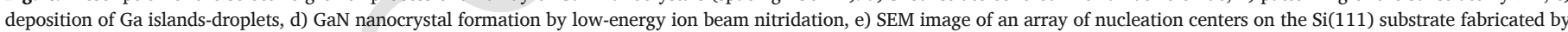
FIB at an ion dose of $6.4 \times 10^{7}$ ions $/ \mu \mathrm{m}^{2}$ for each spot, and f) AFM profiles of the nucleation centers prepared by FIB for different ion doses.

enable us more easily to fabricate arrays of plasmonic antennas attached to these nanocrystals by electron beam lithography (leading to the so called plasmon enhanced photoluminescence). In addition, on purpose built a wide separation of GaN nanocrystals makes it possible to study the optical properties of individual nanocrystals.

The nanocrystals were formed by a sequential deposition, consisting of electron beam evaporation of gallium atoms on Si substrates pre-patterned by FIB and resulting in the Stranski-Krastanov (SK) growth mode followed by post-nitridation by low-energy nitrogen ions $(50 \mathrm{eV})$. Electron beam evaporation is well-matched to the hyperthermal ion beam technique because of the stability and controllability of the deposition process. The combination of these techniques generally provides a higher deposition rate, more uniform coverage, and a cleaner environment than ion beam sputtering.

Observation of photoluminescence (PL) peaks from GaN nanocrystals demonstrates cleanness and quality of the grown crystals. The influence of growth conditions and size of FIB-formed nucleation centers on the properties of GaN nanocrystals is discussed. In addition, we have proposed the method for cultivation of GaN nanocrystals and better control of their size.

\section{Experimental section}

The experiments were performed in a complex UHV apparatus [24] enabling in situ analysis of Ga and GaN structures by X-ray photoelec- 

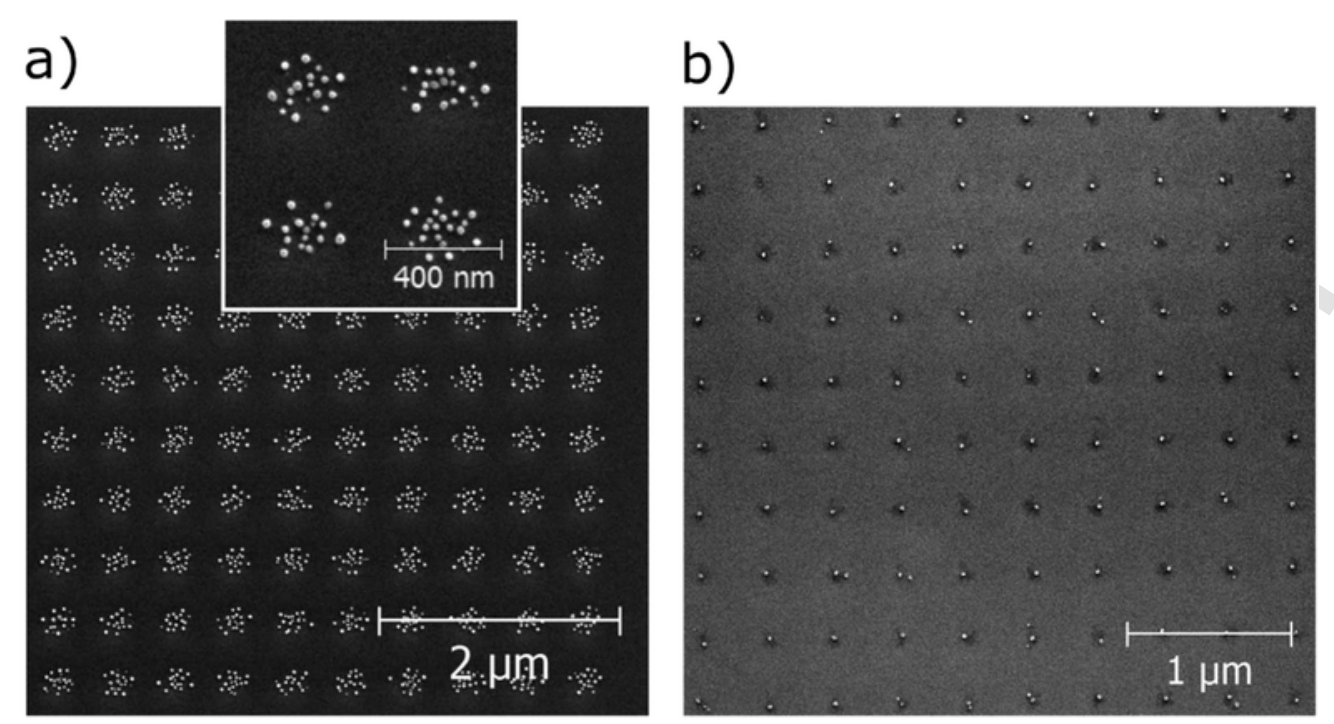

C)

d)
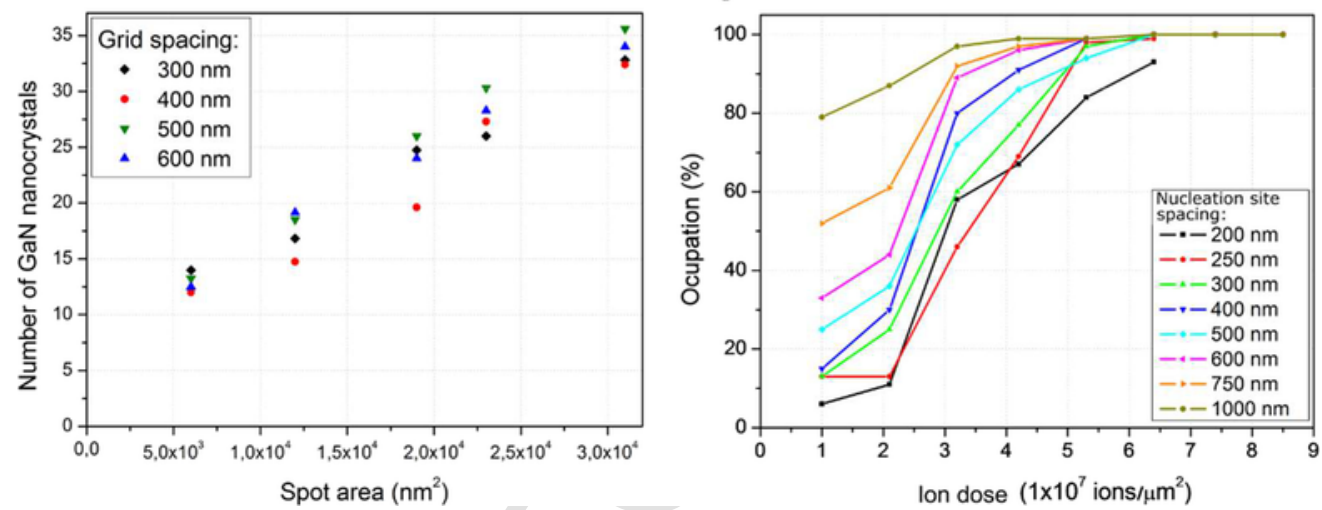

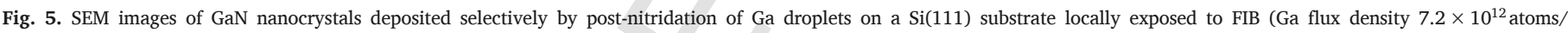

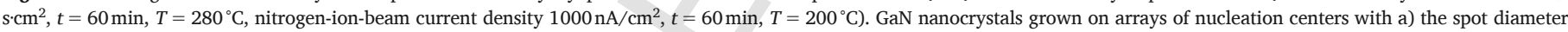

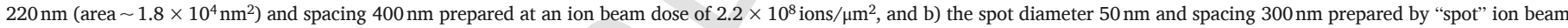

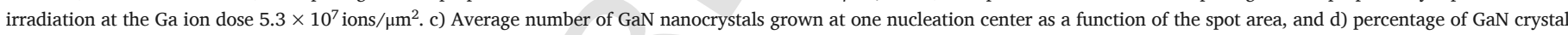
occupation at nucleation sites for different FIB Ga ion doses. Parameter in c) and d): different nucleation site spacing.

tron spectroscopy (XPS) without their exposure to ambient atmosphere. XPS was performed by an experimental setup consisting of the X-ray source - DAR400 and hemispherical electrostatic analyzer - EA125 (both Omicron). Measurements were carried out at room temperature using $\mathrm{Al} \mathrm{K \alpha}$ radiation. The pressure during the measurement was always better than $2 \times 10^{-7} \mathrm{~Pa}$. The morphology was studied ex-situ by scanning electron microscopy (SEM - LYRA 3 system, TESCAN) and atomic force microscopy (AFM - Integra, NT-MDT).

The samples with a size of $5 \times 15 \mathrm{~mm}^{2}$ were cut from a $\mathrm{Si}(111)$ wafer doped by phosphorus (specific resistivity $0.029-0.030 \Omega \cdot \mathrm{cm}$ ). Subsequently, the samples were transferred into the complex UHV apparatus with a base pressure of $3 \times 10^{-8} \mathrm{~Pa}$ and thermally annealed by direct resistive heating at $500^{\circ} \mathrm{C}(>120 \mathrm{~min})$ under UHV conditions. This temperature was sufficient for removal of contaminants from the sample surface, but not high enough for decomposition of the native $\mathrm{SiO}_{2}$ layer [25]. The structure and cleanness of the substrate were checked in situ by XPS.

The substrates were exposed to a gallium atom beam from an effusion cell - (Omicron) and to a ultra-low energy nitrogen ion beam $(50 \mathrm{eV})$ produced from nitrogen gas $(99.9999 \%)$ by a home-made ion-atomic beam source being a combination of an effusion cell and electron impact ion-beam source being $13 \mathrm{~cm}$ away from the sample. The detailed design of the source was described elsewhere [22,23]. The flux of nitrogen ions consists both of $\mathrm{N}^{+}$and $\mathrm{N}^{+}{ }_{2}$ ions (nitrogen current density $j=1000 \mathrm{nA} / \mathrm{cm}^{2}$ ). The work pressure in the deposition chamber measured by an ion gauge during nitridation was $p_{\mathrm{N} 2}=5 \times 10^{-5} \mathrm{~Pa}$. Thermal Ga atoms were evaporated from a PBN boat inside a Mo crucible. The effusion cell was calibrated for a precise control of the $\mathrm{Ga}$ growth rate (Ga flux density $-7.2 \times 10^{12}$ atoms $/ \mathrm{s} \cdot \mathrm{cm}^{2}$ ). The experimental setup is schematically shown in Fig. 1 .

\section{Results and discussion}

\subsection{Formation of GaN nanocrystals}

When Ga atoms and hyperthermal nitrogen ions are simultaneously impinging the surface, they mutually react at the surface and form $\mathrm{Ga}-$ $\mathrm{N}$ bonds. If the ratio of $\mathrm{Ga} / \mathrm{N}_{2}{ }^{+}\left(\mathrm{N}^{+}\right)$flux is set in such a way that each $\mathrm{Ga}$ atom creates a bond with a nitrogen atom on the substrate, potential residual extra nitrogen particles are desorbed and the resulting GaN layer is smooth. This is demonstrated in Fig. 2a, b where SEM images of $\mathrm{GaN}$ layers on a $\mathrm{Si}(111) 7 \times 7$ substrate and native $\mathrm{SiO}_{2}$ surface deposited by a concurrent delivery of $\mathrm{Ga}$ atoms and nitrogen ions at substrate temperatures $T=700^{\circ} \mathrm{C}$ and $T=400^{\circ} \mathrm{C}$, respectively, are shown. 

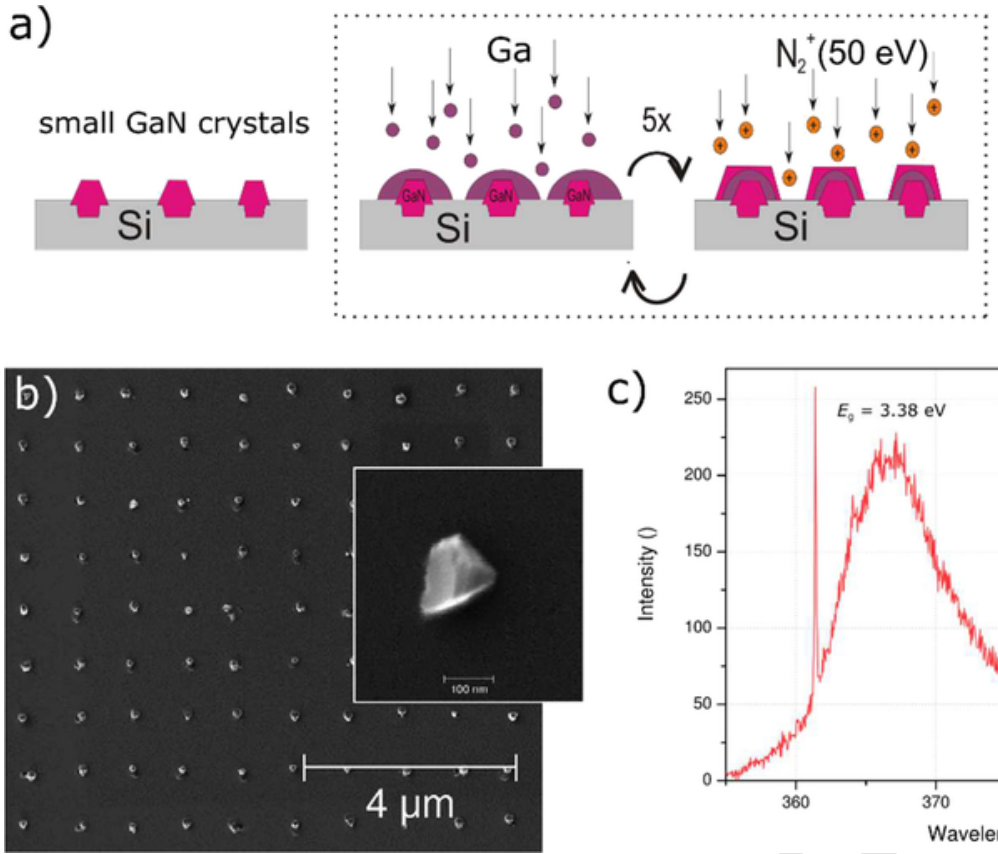

bigger GaN crystals
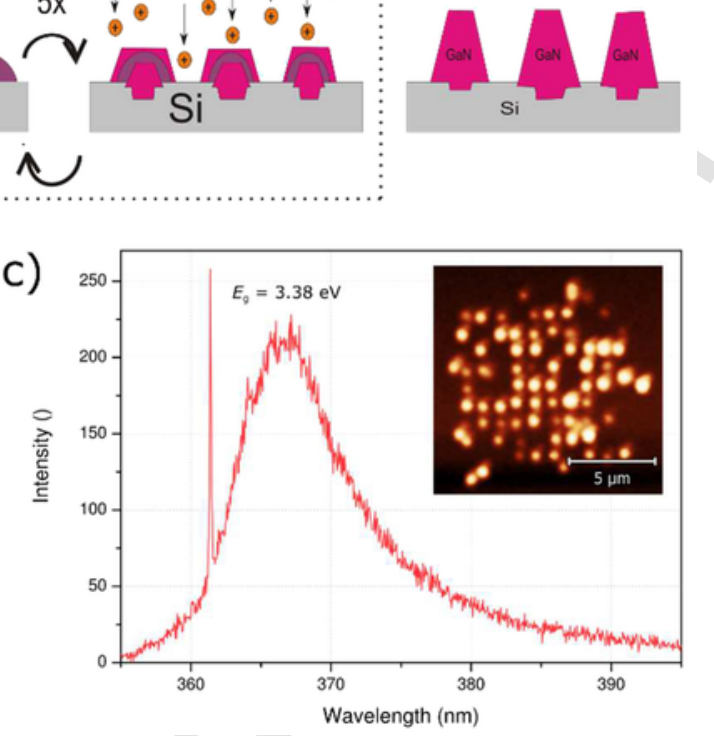

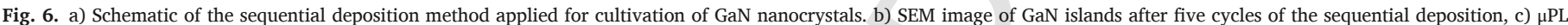
spectrum of ordered GaN crystals after five cycles of the sequential deposition (inset - PL radiation from an array of GaN nanocrystals detected by a CCD camera).

On the other hand, at lower (or zero) fluxes of hyperthermal nitrogen ions, surplus Ga adatoms diffuse along the surface and form Ga droplets at energetically favorable sites, e.g. at terrace edges of the $\mathrm{Si}(111) 7 \times 7$ surface as reported in [23] and shown in Fig. 2c. This is a typical behavior for $\mathrm{Ga}$ atoms as reported in [26]. The size and mutual distance of Ga droplets depend proportionally on substrate temperature and also on surface roughness. In this figure SEM images of GaN smooth layers on terraces of the $\mathrm{Si}(111) 7 \times 7$ substrate with an excess of $\mathrm{Ga}$ atoms condensed preliminary in form of droplets at edges of terraces are shown (Ga flux density - $2.6 \times 10^{16}$ atoms $/ \mathrm{h} \cdot \mathrm{cm}^{2}$, nitrogen current density $j=1000 \mathrm{nA} / \mathrm{cm}^{2}$, substrate temperature $T=300^{\circ} \mathrm{C}$ ). It is worth mentioning that some droplets already contain nuclei of $\mathrm{GaN}$ at the interface with the substrate as can be seen in detail in Fig. 2d. It is in agreement with [27] where it is hypothesized that the top surface of liquid Ga droplets acts as a major mass transport path for nitrogen species delivered by hyperthermal nitrogen ions which then diffuse to peripheral regions of the droplet-substrate interface. Hence, this interface acts as a collector of nitrogen particles dissolved on the droplet (and of those diffusing towards the interface from the substrate surface around droplets) as schematically depicted in Fig. 3. These species initiate formation of $\mathrm{Ga}-\mathrm{N}$ crystallization nuclei at the liquid-solid interface, which is followed by propagation of a crystallization process from the peripheral droplet region towards the center of the interface.

In Fig. 2e a SEM image of Ga droplets deposited without nitrogen ion flux for $60 \mathrm{~min}$ on the native $\mathrm{SiO}_{2}$ surface by a $\mathrm{Ga}$ atomic flux density of $7.2 \times 10^{12}$ atoms $/ \mathrm{s} \cdot \mathrm{cm}^{2}$ at substrate temperature $T=200^{\circ} \mathrm{C}$ is shown. The image of $\mathrm{Ga}$ droplets exposed for $60 \mathrm{~min}$ to the current of low energy nitrogen ions $\left(j=1000 \mathrm{nA} / \mathrm{cm}^{2}\right)$ at substrate temperature $T=200{ }^{\circ} \mathrm{C}$ can be seen in Fig. 2f. In the figure the formation of crystals is apparent. Their chemical composition was checked by XPS which proved the crystals consisted of GaN. It is worth noting that the transformation of $\mathrm{Ga}$ islands into $\mathrm{GaN}$ nanocrystals was carried out without breaking UHV conditions.

The size and quantity of Ga droplets enabling transformation to GaN crystals depend on the sticking coefficient of Ga atoms on the substrate [26] which is a function of substrate temperature.
At higher temperatures, Ga droplets do not nucleate because most of gallium atoms do not stick to the surface (e.g. $T>450^{\circ} \mathrm{C}$ on $\mathrm{Si}(111) 7 \times 7$ or $\mathrm{T}>300^{\circ} \mathrm{C}$ on $\mathrm{SiO}_{2}$ ) and the substrate is covered only by a GaN layer formed by instantaneous local interaction of $\mathrm{Ga}$ atoms with nitrogen species just upon their impingement on the surface. Thus, the final morphology of the ultrathin film prepared by the MBE and direct ion beam deposition is given by a combination of two events: on the one hand, by a direct impact of a nitrogen ion into close vicinity of a Ga adatom (smooth layer), and on the other hand, by its impact on a Ga island (nanocrystal). Consequently, smoother films can be grown by a suitable setting of the $\mathrm{Ga}$ atom/nitrogen ion impact ratio. On the other hand, single GaN nanocrystals can be formed by a separate deposition of $\mathrm{Ga}$ atoms first, followed by a subsequent nitrogen ion beam irradiation (energy of ions below $50 \mathrm{eV}$ ).

\subsection{Selective growth of GaN nanocrystals}

The surface diffusion plays an important role during selective growth of various materials, as atoms of deposited materials have to be transported to nucleation sites [9]. However, at temperatures in the range from RT to $800^{\circ} \mathrm{C}$ the surface diffusion length of $\mathrm{Ga}-\mathrm{N}$ dimers on $\mathrm{SiO}_{2}$ and Si surfaces is significantly shorter than for $\mathrm{Ga}$ atoms on these surfaces. This shorter diffusion length does not allow carrying out the selective growth by the simultaneous deposition of Ga atoms and nitrogen ions. Under the conditions of this concurrent deposition the SK growth mode has not been observed for $\mathrm{SiO}_{2}$ surfaces and $\mathrm{Si}(111) 7 \times 7$ substrates in the temperature range RT $-600^{\circ} \mathrm{C}$ and $\mathrm{RT}-800^{\circ} \mathrm{C}$, respectively. Hence, when the $\mathrm{Ga}$ atom/nitrogen ion flux ratio is set at these temperatures to the values not leading to the nucleation of $\mathrm{Ga}$ islands, the growth results in a smooth GaN layer as shown in Fig. 2a and b.

To achieve the selective growth of ordered GaN nanostructures, a two-step deposition procedure described already above was applied. In the first step, $\mathrm{Ga}$ atoms were deposited on a surface with nucleation sites created by FIB. Due to a long surface diffusion length Ga atoms were transported towards these nucleation sites and formed droplets there. During the second step, Ga droplets were transformed into GaN 
nanocrystals by low energy nitrogen ions $(50 \mathrm{eV})$ impinging upon them. Such a mechanism of the sequential deposition method enabling fabrication of ordered self-assembled arrays of GaN nanocrystals by this post-nitridation process is schematically shown in Fig. 4a-d.

The experiments were carried out on $10 \times 10$ arrays of circular nucleation sites of a diameter ranging from $100 \mathrm{~nm}$ to $300 \mathrm{~nm}$ and produced by respective ion doses $1.1 \times 10^{7}$ ions $/ \mu \mathrm{m}^{2}-31 \times 10^{7} \mathrm{ions} / \mu \mathrm{m}^{2}$. The spacing of the nucleation centers varied from $100 \mathrm{~nm}$ to $1500 \mathrm{~nm}$ on the $\mathrm{Si}(111)$ substrate covered with a $2 \mathrm{~nm}$-thick native $\mathrm{SiO}_{2}$ layer. A typical FIB-patterned substrate surface before the nucleation of $\mathrm{Ga}$ atoms is shown in Fig. 4e. The profiles of nucleation centers fabricated by FIB at different Ga ion doses measured by AFM are shown in Fig. 4f.

First, at lower ion beam doses the sub-surface layers are amorphized and implanted by Ga ions, which leads to creation of conical features swollen above the surface. It is only for ion beam doses above $5.39 \times 10^{-7}$ ions $\cdot \mathrm{mm}^{-2}$ when the central hole - crater in these features is observed.

Ga atoms were deposited on the FIB patterned substrate at the substrate temperature $T=280^{\circ} \mathrm{C}$ and a flux density of $7.2 \times 10^{12}$ atoms/ $\mathrm{s} \cdot \mathrm{cm}^{2}$ for $60 \mathrm{~min}$. As the sticking coefficient of $\mathrm{Ga}$ atoms on the flat $\mathrm{SiO}_{2}$ surface is close to zero [9], Ga atoms nucleated preferentially at the surface defects fabricated by FIB. Subsequently, Ga islands were exposed to a low energy $(50 \mathrm{eV})$ nitrogen ion beam with the current density $1000 \mathrm{nA} / \mathrm{cm}^{2}$ for $60 \mathrm{~min}$ at the substrate temperature $200^{\circ} \mathrm{C}$. After this procedure GaN crystals occupied positions exposed to FIB only.

The typical size of deposited crystals measured by contact-AFM was in the range $20-40 \mathrm{~nm}$. The number of GaN crystals occupying the milled nucleation sites depends on the area of these nucleation centers - further called the spot areas (defined as the inner area of the milled crater). Fig. 5a shows GaN crystals grown selectively at circularly shaped nucleation centers of an area of $1.8 \times 10^{4} \mathrm{~nm}^{2}$ and grid spacing of $400 \mathrm{~nm}$ prepared by ion-beam scanning over a disk-like area of a diameter of $220 \mathrm{~nm}$. The number of GaN crystals located inside the FIB - made nucleation site depends linearly on its area as shown for different spacing of nucleation sites $(300-600 \mathrm{~nm})$, in Fig. 5c. From the results depicted in this figure one cannot claim that decreasing the spot size leads to 1 nanocrystal occupancy. To achieve just one nanocrystal at a FIB-made nucleation center one should apply the so-called "spot" ion-beam irradiation where the ion beam is fixed to one spot without scanning over the time An example of typical selectively deposited GaN crystal structures mostly possessing one GaN crystal in each nucleation site and prepared by this method at an ion beam dose of $5.3 \times 10^{7} \mathrm{ions} / \mu \mathrm{m}^{2}$ for an array spacing of $300 \mathrm{~nm}$ is shown in Fig. $5 \mathrm{~b}$.

The occupation of nucleation sites by GaN nanocrystals depends on the FIB Ga ion dose and on the spacing of nucleation sites as well, as shown in Fig. 5d. The higher dose of Ga ions has a more profound influence on the nucleation site profile (see Fig. 4f) and thus the crater area, which leads to the higher capture probability of Ga atoms at this site. At the same time, the occupation probability of the nucleation sites made by FIB rises with the increasing spacing of nucleation sites. This is caused by an increase of the collection area of $\mathrm{Ga}$ atoms related to one nucleation site (a decrease of the Ga depletion effect). Obviously, an increase of the nucleation site occupation at a lower spacing of nucleation sites can be provided by a higher $\mathrm{Ga}$ atom coverage on the surface.

\subsection{Cultivation of nanocrystals}

The main challenge of the post-nitridation method is the preparation of $\mathrm{GaN}$ nanocrystals with a size bigger than $40 \mathrm{~nm}$. The size of these nanocrystals is primarily controlled by the dimension of Ga droplets. Generally, the size of Ga nanodroplets should grow with the substrate temperature. However, to ensure sufficient mobility of $\mathrm{Ga}$ atoms to reach comfortably pre-patterned confining spots, the substrate temperature must be kept close to the values where the sticking coefficient of $\mathrm{Ga}$ on the $\mathrm{SiO}_{2}$ substrate is nearly zero $\left(\sim 280^{\circ} \mathrm{C}\right)$. Hence, there is a sufficient depletion of the Ga material feeding the spots and $\mathrm{Ga}$ droplets remain small.

To overcome this problem, we have proposed a method schematically depicted in Fig. 6a. It is based on the already discussed sequential deposition being repeatedly applied in several cycles. In the first step, small GaN crystals are formed by post-nitridation of Ga droplets and then further sequences of Ga deposition and Ga post-nitridation are performed for several times. In this way, the crystals can be significantly increased as demonstrated in Fig. $6 \mathrm{~b}$ for the case of five deposition cycles.

In the first step of this experiment, small GaN crystals were formed by the sequential deposition at conditions described in Section 3.2. After that, the first sequential deposition cycle carried out under slightly distinct conditions started - In this cycle gallium was deposited on the sample for $60 \mathrm{~min}$ at $300^{\circ} \mathrm{C}$ and a Ga flux density of $7.2 \times 10^{12}$ atoms/ $\mathrm{s} \cdot \mathrm{cm}^{2}$. A bit increased substrate temperature in this step towards the previous one (i.e. $300^{\circ} \mathrm{C}$ to $280^{\circ} \mathrm{C}$ ) provided enhanced surface diffusion of $\mathrm{Ga}$ atoms and their nucleation at small GaN nanocrystals. Then the surface was cooled down to $200^{\circ} \mathrm{C}$ and exposed for $60 \mathrm{~min}$ to the $50 \mathrm{eV}$ nitrogen ion beam with a current density of $1000 \mathrm{nA} / \mathrm{cm}^{2}$ to accomplish post-nitridation. Such a deposition cycle was repeated for five times.

In the inset of Fig. $6 \mathrm{~b} \mathrm{GaN}$ crystal facets are clearly visible. To check the quality of GaN crystals, photoluminescence properties of GaN crystals grown up to an approximate size of $150 \mathrm{~nm}$ and $200 \mathrm{~nm}$ (measured by AFM) were tested.

The PL measurement was carried out at room temperature using a micro-Raman spectroscopy system (WITEC, alfa 300R) with the excitation laser wavelength $\lambda_{\mathrm{ex}}=355 \mathrm{~nm}$ (solid state laser). The PL emission was detected by a CCD camera (DU420A-BU-352) and spectra taken by a spectrophotometer (UHT 400). The diameter of the focused laser spot was about $0.5 \mu \mathrm{m}$.

A typical PL spectrum obtained for a laser excitation power of $2 \mathrm{~mW}$ is demonstrated in Fig. 6c. The spectrum was collected from an array of GaN nanocrystals shown in the inset of the figure and clearly revealed the peak of a band edge emission at around $367 \mathrm{~nm}(3.38 \mathrm{eV}$, blue luminescence). Interestingly, not all nanocrystals were PL active despite their similar morphology and size. With this respect, additional studies going beyond the scope of this paper should be carried out.

\section{Conclusion}

The successful approach on UHV growth of arrays of GaN nanocrystals has been presented. This selective growth was achieved by replacing a concurrent deposition of Ga atoms and nitrogen ions, provided by the simultaneous MBE and direct ion beam deposition and preferentially leading to continuous thin films of GaN, by time splitting of delivery of these individual constituents on a FIB pre-patterned $\mathrm{SiO}_{2}$ substrate. It means, first $\mathrm{Ga}$ atoms were deposited on these pre-patterned substrate at a trade-off temperature $\left(280^{\circ} \mathrm{C}\right)$ high enough to reach the FIB-made nucleation spots and sufficiently low to prevent desorption of $\mathrm{Ga}$ atoms from the surface. After that a post- nitridation of small Ga droplets nucleated at the spots was carried out by a $50 \mathrm{eV}$-nitrogen ion beam deposition to get GaN nanocrystals at $T=200^{\circ} \mathrm{C}$. Synthesis of $\mathrm{GaN}$ at such a low temperature was achieved by an excess energy locally delivered by hyperthermal nitrogen ions. To get larger arrays of GaN nanocrystals $(\approx 150 \mathrm{~nm}$ and $200 \mathrm{~nm}$ in diameter), such a sequential deposition was repeated in several cycles at slightly modified operation conditions. The quality of the nanocrystals was checked by measurement of their photoluminescence properties which proved the occurrence of the peak of a band edge emission at around $367 \mathrm{~nm}(3.38 \mathrm{eV})$. 


\section{Acknowledgment}

This work was supported by the Technology Agency of the Czech Republic (grant No. TE01020233), H2020 Twinning programme (project SINNCE, 810626), Grant Agency of the Czech Republic (grant 17-33767L), and MEYS CR (grant No. LQ1601 - CEITEC 2020). We also acknowledge the support by the Grant Agency of the Czech Republic (grant No. 17-21413S).

\section{References}

[1] C. Lee, A.L. Barabási, Spatial ordering of islands grown on patterned surfaces, Appl. Phys. Lett. 73 (18) (1998) 2651-2653.

[2] T.W.H. Oates, A. Keller, S. Noda, S. Facsko, Self-organized metallic nanoparticle and nanowire arrays from ion-sputtered silicon templates, Appl. Phys. Lett. 93 (6) (2008) 063106-3.

[3] K.V. Sarathlal, D. Kumar, A. Gupta, Growth study of Co thin film on nanorippled Si(100) substrate, Appl. Phys. Lett. 98 (12) (2011) 123111-123113.

[4] V. Repain, J.M. Berroir, S. Rousset, J. Lecoeur, Growth of self-organized cobalt nanostructures on Au(111) vicinal surfaces, Surf. Sci. 447 (1) (2000) L152-L156.

[5] B. Yang, F. Liu, M.G. Lagally, Local strain-mediated chemical potential control of quantum dot self-organization in heteroepitaxy, Phys. Rev. Lett. 92 (2) (2004) 025502-025504.

[6] H. Sekiguchi, K. Kishino, A. Kikuchi, Ti-mask selective-area growth of GaN by RF-plasma-assisted molecular-beam epitaxy for fabricating regularly arranged InGaN/GaN nanocolumns, Appl. Phys. Express 1 (12) (2008) 124002-124003.

[7] H. Sekiguchi, K. Kishino, A. Kikuchi, Emission color control from blue to red with nanocolumn diameter of $\mathrm{InGaN} / \mathrm{GaN}$ nanocolumn arrays grown on same substrate, Appl. Phys. Lett. 96 (23) (2010) 96-99.

[8] L. Liu, M. Shao, L. Cheng, S. Zhuo, R. Que, S.T. Lee, Edge-enhanced Raman scattering effect from Au deposited nanoedge array, Appl. Phys. Lett. 98 (7) (2011) 073114-3.

[9] M. Bartosík, M. Kolíbal, J. Cechal, J. Mach, T. Sikola, Selective growth of metallic nanostructures on surfaces patterned by AFM local anodic oxidation, J. Nanosci. Nanotechnol. 9 (10) (2009) 5887-5890.

[10] T. Yasuda, S. Yamasaki, Nanoscale selective-area epitaxial growth of Si using an ultrathin $\mathrm{SiO}_{2} / \mathrm{Si}_{3} \mathrm{Ni}_{4}$ mask patterned by an atomic force microscope, Appl. Phys. Lett. 77 (24) (2000) 3917-3919.
[11] T. Mårtensson, P. Carlberg, M. Borgström, L. Montelius, W. Seifert, L. Samuelson, Nanowire arrays defined by nanoimprint lithography, Nano Lett. 4 (4) (2004) 699-702.

[12] X. Qian, J. Li, D. Wasserman, W.D. Goodhue, Uniform InGaAs quantum dot arrays fabricated using nanosphere lithography, Appl. Phys. Lett. 93 (23) (2008) 1-4.

[13] K. Meneou, K.Y. Cheng, Z.H. Zhang, C.L. Tsai, C.F. Xu, K.C. Hsieh, Site-controlled InAs quantum dots regrown on nonlithographically patterned GaAs, Appl. Phys. Lett. 86 (15) (2005) 1-3.

[14] J. Tommila, et al., Nanoimprint lithography patterned GaAs templates for site-controlled InAs quantum dots, J. Cryst. Growth 323 (1) (2011) 183-186.

[15] C.C. Cheng, K. Meneou, K.Y. Cheng, Effects of nano-pattern size on the property of InAs site-controlled quantum dots, J. Cryst. Growth 323 (1) (2011) 180-182.

[16] E. Sel̦uk, A.Y. Silov, R. Nötzel, Single InAs quantum dot arrays and directed self-organization on patterned GaAs (311)B substrates, Appl. Phys. Lett. 94 (26) (2009) 263108-3.

[17] H. Heidemeyer, C. Müller, O.G. Schmidt, Structural and optical investigations of 1-, 2-, and 3-dimensional InAs quantum dot arrays, Physica E 23 (3-4) (2004) $237-242$.

[18] S.D. Hersee, J.C. Ramer, K.J. Malloy, G. Sapphire, The Microstructure of Metalorganic-Deposition GaN on Sapphire, no. July 199745-51.

[19] C. Skierbiszewski, et al., Nitride-based laser diodes grown by plasma-assisted molecular beam epitaxy, J. Phys. D. Appl. Phys. 47 (7) (2014) 073001-073018.

[20] M. Kappers, J.L. Guyaux, J. Olivier, R. Bisaro, C. Grattepain, J.C. Garcia, Chemical beam epitaxy of GaN on (0001) sapphire substrate, Mater. Sci. Eng. B 59 (1-3) (1999) 52-55.

[21] T. Nagata, P. Ahmet, T. Sekiguchi, T. Chikyow, Low-temperature growth of GaN microcrystals from position-controlled Ga droplets arrayed by a low-energy focused ion beam system, J. Cryst. Growth 283 (3-4) (2005) 328-331, Oct.

[22] J. Mach, et al., An ultra-low energy (30-200 eV) ion-atomic beam source for ion-beam-assisted deposition in ultrahigh vacuum, Rev. Sci. Instrum. 82 (8) (2011) 083302-083308.

[23] J. Mach, et al., Optimization of ion-atomic beam source for deposition of GaN ultrathin films, Rev. Sci. Instrum. 85 (8) (2014) 083302-083306.

[24] S. Voborný, et al., Deposition and in-situ characterization of ultra-thin films, Thin Solid Films 459 (1-2) (2004) 17-22, Jul.

[25] K. Xue, J.B. Xu, H.P. Ho, Nanoscale in situ investigation of ultrathin silicon oxide thermal decomposition by high temperature scanning tunneling microscopy, Nanotechnology 18 (48) (2007) 485709-7.

[26] M. Kolíbal, T. Čechal, E. Brandejsová, J. Čechal, T. Šikola, Self-limiting cyclic growth of gallium droplets on Si(111), Nanotechnology 19 (47) (2008) 475606-5.

[27] J.W. Gerlach, T. Ivanov, L. Neumann, T. Höche, D. Hirsch, B. Rauschenbach, Epitaxial GaN films by hyperthermal ion-beam nitridation of Ga droplets, J. Appl. Phys. 111 (11) (2012) 113521-10. 